\title{
MENDICIDAD, ABANDONO Y TRABAJO INFANTIL EN LA COMUNIDAD GARÍFUNA DE TRAVESÍA, PUERTO CORTES
}

\section{Begging, abandonment and child labor in the comunidad garifuna de Travesia, Puerto Cortes}

\author{
*Vilma Mercedes Miranda Baquedano, **Lesby Melissa Flores Girón, \\ **Erick Alejandro Euceda Garza.
}

\section{RESUMEN}

La mendicidad, abandono y trabajo infantil son una realidad social en la que se ven involucrados muchos niños y niñas, siendo estos una población vulnerable ante los factores que potencian esta situación Objetivo: Conocer la prevalencia de mendicidad, abandono y trabajo infantil en la comunidad Garífuna de Puerto Cortés, I semestre 2016. Pacientes y Métodos: Estudio cuantitativo, descriptivo/exploratorio, transversal. Población 450 familias, muestra 149 familias, aleatorio simple. Instrumento tipo cuestionario. Resultados: $47 \%$ de las personas han observado niños mendigando, $86.58 \%$ si sabe que es abandono, $87.2 \%$ no conoce instituciones que ayuden a los niños abandonados, en $42.28 \%$ de los hogares habitan niños abandonados por algún familiar, $50.79 \%$ indicaron ser la abuela de los niños. $26.17 \%$ de los hogares los menores realizan actividades para generar ingresos económicos, $13.51 \%$ realizan ventas ambulantes y el $4.73 \%$ construcción. Conclusión: Los resultados de la investigación muestran que si hay abandono, tanto físico como emocional en los niños de la comunidad Garífuna, al igual que la mendicidad, aunque no se observan niños pidiendo a simple vista estos si rodean la comunidad o realizan esta actividad durante las fechas donde hay más turistas, el trabajo infantil no se ve muy marcado en la población encuestada, sin embargo los niños si realizan actividades económicas para

*Profesora de la Carrera de Enfermería de la Escuela Universitaria de Ciencias de la salud (EUCS) de la Universidad Nacional Autónoma de Honduras en el Valle de Sula (UNAH -VS). Licenciada en Enfermería. Especialista en Salud Materno Perinatal. Magister en Gestión Educativa.

** Estudiantes de la Carrera de Enfermería de la EUCS-UNAHVS.

Dirigir correspondencia a: vilmamiranda2012@yahoo.es

Recibido: 15 de septiembre 2016 Aprobado: 20 de diciembre 2016. generar ingresos en su hogar, como la venta de pan de coco, tabletas, algunos en construcción, la mayoría de estos niños trabajan también estudian.

\section{PALABRAS CLAVE}

Pobreza, niño abandonado, trabajo infantil, grupo étnico, niños.

\section{ABSTRACT}

Begging, abandonment and child labor are a social reality in which they are involved many boys and girls, being these population vulnerable to factors that enhance this situation. Objective: Know the prevalence of begging, abandonment and child labor in the Garífuna community of Puerto Cortes first semester 2016. Patients and Methods: Quantitative study non-experimental, cross- sectional, descriptive/exploratory population 450 homes, displays 149 families, Instrument type: questionnaire. Results: $47 \%$ people have observed children begging in the community, the $86.58 \%$ of the population do know what the abandonment is, $87.2 \%$ don't know institutions within the community that help children abandoned, in the $42.28 \%$ of the homes do live children aside from their children, the $50.79 \%$ indicated to be the grandmother of the children, in the $26.17 \%$ percent of homes the children do make activities to generate income, the $13.51 \%$ make hawking, $4.73 \%$ construction. Conclusion: The results of the investigation show that there is abandonment, both physical and psychologically on the children of the Garifuna community, such as begging, although children are not observed that request in the light but these do surround the community or to carry out this activity during the dates 
where there are more tourists, child labor is not very marked in the surveyed population, however the children do carry out economic activities to generate income in their homes, such as the sale of coco bread, tablets, some work in construction, most of these children that work they also study.

\section{KEYWORDS}

Poverty, Child Abandoned, child labor, ethnic group, children.

\section{INTRODUCCIÓN}

La mendicidad, el abandono y el trabajo infantil son una realidad social donde se ven involucrados muchos niños y niñas, siendo estos una población vulnerable ante los diversos factores que potencian esta situación. Actualmente la población infantil de Honduras es aproximadamente 3,6 millones, 1,9 son del sexo masculino $(53 \%)$ y 1,2 millones son del sexo femenino (47\%). ${ }^{(1)}$ De acuerdo a instituciones estatales más del $50 \%$ de la población hondureña vive bajo la denominada línea de la pobreza, razón por la cual tenemos un $29.1 \%$ de niños y niñas viviendo en situación de extrema pobreza, con un margen de diferencia muy notorio entre la población infantil de la zona rural $(40.4 \%)$ y la de la zona urbana $(12.7 \%){ }^{(1)}$

No existen datos estadísticos específicos sobre la situación de mendicidad y abandono en las comunidades Garífunas de Honduras, sin embargo, según la Encuesta del Estado de la Niñez Indígena y Afrohondureña (2011), 3.8\% de la población infantil Garífuna de 6-17 años trabaja fuera del hogar, siendo el $2.9 \%$ niños y $4.8 \%$ niñas". ${ }^{(2)}$ Un estudio específico de la OIT (Organización Internacional del Trabajo) indica que la población infantil indígena y afrohondureña realiza trabajos nocivos: "la ubicación geográfica de las comunidades y las principales fuentes de trabajo existentes definen las condiciones a las que se enfrentan estos niños, niñas y adolescentes indígenas y afrodescendientes: trabajos duros, mal pagados, poco cualificados, con escasa repercusión en la economía nacional y nula promoción interna". (2)
MENDICIDAD: es un fenómeno asociado a la pobreza, la indigencia, el desempleo, la miseria y en fin a la falta de los recursos necesarios de una gran parte de la población, para proveerse de la subsistencia. ${ }^{(3)}$ No tienen elección, han sido abandonados, son huérfanos o han sido expulsados de sus hogares, viven en la calle a raíz de maltratos sufridos en su casa, por negligencia de los padres, desintegración del entorno familiar, pobreza extrema desastres (naturales o provocados), hambre, abusos físicos y sexuales, explotación infantil, desplazamiento social tras emigración. ${ }^{(3)}$ Algunas de las causas pueden ser: maltrato infantil, falta de recursos económicos, autonomía económica, explotación por parte de personas adultas, falta de autoridad paterna y/o materna (familia disfuncional), falta de educación en los padres, problemas sociales y analfabetismo. Según Fernanda Zumárraga "Los niños o niñas se ven obligados a la realización continua de actividades o acciones consistente en demandas o pedido de dinero en la vía pública, este tipo de maltrato se encuentra muy relacionado con la explotación laboral ya que son asignados a los mismos con el objeto de obtener un beneficio económico, sin tener que realizar, los adultos ninguna otra tarea".(4)

ABANDONO INFANTIL: también llamado maltrato psicológico, es la falta de atención a las necesidades básicas de un niño, es decir, ocurre cuando alguien intencionalmente no le suministra al niño alimento, agua, vivienda, vestido, atención médica u otras necesidades. Existen cuatro tipos: el abandono físico es una supervisión inadecuada y/o poco segura del niño, el abandono médico es negarle al niño la atención médica que necesite o un tratamiento médico que se le haya prescrito, el cual podría incluir nutrición, hidratación y medicación apropiadas, el abandono educativo es el incumplimiento de las leyes del estado respecto a educación infantil obligatoria y el abandono emocional es ignorar las necesidades del niño para poder tener un desarrollo social y emocional normal. ${ }^{(5)}$ Todas estas actividades realizadas que conforman el abandono infantil son una problemática muy 
latente en las sociedades y no conoce límites fronterizos, culturales ni económicos, está muy marcado y como consecuencia existen ambientes familiares conflictivos principalmente que llevan a la destrucción familiar y a su vez a la desorientación social del niño en este caso siendo el más afectado. Puede afectar al grado de incurrir en un desequilibrio de su salud, logra atentar contra la seguridad de este y lo percibe con un constante desprecio hacia los miembros de la familia en un inicio y a futuro por parte del menor que se convierte en una problemática no solamente familiar sino de la sociedad porque los niños/niñas son el futuro.

TRABAJO INFANTIL: según la OIT el trabajo infantil es aquél que priva a los niños de vivir su etapa de niñez de manera natural. Son las tareas que les impiden desarrollarse adecuadamente y de manera digna. ${ }^{(6)}$

Es importante tener en cuenta la cantidad de horas a las que el niño está sometido a realizar la tarea, al igual que los objetivos que hay detrás de la misma. También dentro de la niñez hay un rango de edades, y no es lo mismo un niño de 4 años realizando un trabajo duro, que uno de once vendiendo limonada. ${ }^{(6)}$ No les permite escolarizarse como corresponde, compromete su integridad física y su salud mental y afecta sus relaciones personales. ${ }^{(6)}$

El trabajo infantil se ha visto fuera de control en los países en vías de desarrollo donde hasta cierto punto es aceptado, volviéndose una situación alarmante ya que en esta etapa existe un mayor riesgo de su condición de salud, alterando su desarrollo fisiológico. Al contrario en países con desarrollo social, económico, educativo, etc., esta actividad es totalmente prohibida y penada y hay compromiso de mantener un ambiente seguro y apto para el completo bienestar y desarrollo de los niños donde estos tendrán su oportunidad de realizar actividades laborales en su determinado momento y de acuerdo a su etapa fisiológica.

LOS GARÍFUNAS: desde el siglo XVIII, es evidente la existencia de un número importante de negros, mulatos y pardos, que se encuentran asentados a lo largo del territorio en Guatemala, Nicaragua. Además de los pueblos de San Vicente en El Salvador y La Puebla de los Pardos en Costa Rica, y en el Atlantico Hondureño por citar solo algunos. ${ }^{(7)}$

El pueblo Garífuna, es uno de los grupos étnicos más grande que habita en las costas de Honduras con una población aproximada de 300,000 habitantes. Están distribuidos en 47 comunidades ubicadas a lo largo de la costa Norte Hondureña, específicamente en los departamentos de Cortés, Atlántida, Colón, Gracias a Dios e Islas de La Bahía. ${ }^{(8)}$ En las características socioeconómicas existe un espíritu igualitario en la mayoría de las comunidades étnicas. Sin embargo en actividades como la construcción de vivienda, la pesca y la elaboración del casabe la práctica de la ayuda mutua está bastante debilitada. ${ }^{(9)}$ El $54 \%$ de la población Garífuna posee títulos ejidales y el resto posee tierras comunales que en la mayoría de los casos enfrentan conflictos de usurpación con inmigrantes que llegan con el propósito de instalar haciendas ganaderas, agroindustrias o empresas turísticas. ${ }^{(9)}$

Objetivo Conocer la prevalencia de mendicidad, abandono y trabajo infantil en la comunidad Garífuna Travesía de Puerto Cortés en el 1 semestre del 2016.

\section{MATERIALESY METODOS}

Se realizó un estudio cuantitativo, exploratorio/descriptivo, transversal, con el propósito de conocer la prevalencia de mendicidad, abandono y trabajo infantil de la comunidad garífuna de Travesía, Puerto Cortés. El universo fue de 450 familias, tomando una muestra probabilística (aleatorio simple) 184 familias previo consentimiento informado, de las cuales se encuestaron 149 debido a diferentes inconvenientes que impidieron la captación total de la muestra como ser, viviendas cerradas y personas renuentes a participar. Se realizó una 
encuesta que contaba con 31 preguntas abiertas y cerradas, en las cuales se identificaron las 3 principales categorías de la investigación; mendicidad, abandono y trabajo Infantil. Los datos fueron analizados utilizando el programa EPI INFO 7.1.5.2.

\section{RESULTADOS}

De las 149 personas encuestadas $8.8 \%$ tenían rango de edad de 15-19 años, 12\% tenían de 20-24 años, $16.1 \%$ de $25-29$ años, $16.8 \%$ de 30-34 años, $10 \%$ 35-39 años, el $16.1 \%$ de $40-49$ años, $7.4 \%$ de $50-59$ y $12.8 \%$ de 60 o más.

El $25.5 \%$ de las personas tenían primaria completa, $25.5 \%$ primaria incompleta, $29.5 \%$ tenía secundaria incompleta, $14.8 \%$ secundaria completa, $2 \%$ ninguno y $0.7 \%$ culminó la Universidad y $2 \%$ universidad incompleta.

Según la ocupación de las personas encuestadas, $76.5 \%$ eran amas de casa, $3.4 \%$ mecánico, $2.7 \%$ albañil, 3.4\% comerciante, 3.4\% estudiante, $2 \%$ operaria, $1.3 \%$ ebanista, $1.3 \%$ carpintero, 6\% otros. El ingreso mensual de las familias es menos del salario mínimo $64.4 \%$, el $20.1 \%$ salario mínimo, el $8.1 \%$ no sabe y el $7.4 \%$ más del salario mínimo.

Los niños dentro de las familias encuestadas tenían estos rangos de edad. (Ver Tabla No.1.)

TABLA No. 1: Edades de los niños de las familias encuestadas.

\begin{tabular}{|c|c|c|}
\hline Edad & Frecuencia & Porcentaje \\
\hline $\begin{array}{c}1--11 \\
\text { meses }\end{array}$ & 21 & $6 \%$ \\
\hline $1-3$ años & 67 & $19 \%$ \\
\hline $4-6$ años & 64 & $18 \%$ \\
\hline 7-9 años & 60 & $17 \%$ \\
\hline $10-12$ años & 51 & $14 \%$ \\
\hline $13-15$ años & 48 & $13 \%$ \\
\hline $16-18$ años & 47 & $13 \%$ \\
\hline Total & 358 & $100 \%$ \\
\hline
\end{tabular}

Fuente: Encuesta Asignatura Familiar II
Los escolares habían cursado los siguientes grados: $8.1 \%$ kinder, $9.2 \%$ primer grado, $6.7 \%$ tercer grado, $7.5 \%$ cuarto grado, $5.6 \%$ quinto grado, $8.4 \%$ octavo grado, $6.1 \%$ noveno grado, $5.3 \%$ bachillerato, $28.2 \%$ ninguno y $14.9 \%$ son bebes y menores que no estaban en edad escolar.

Las personas encuestadas han observado niños mendingando. (Ver Grafica No. 1.)

Grafica No. 1: Ha Observado niños mendigando en la comunidad.

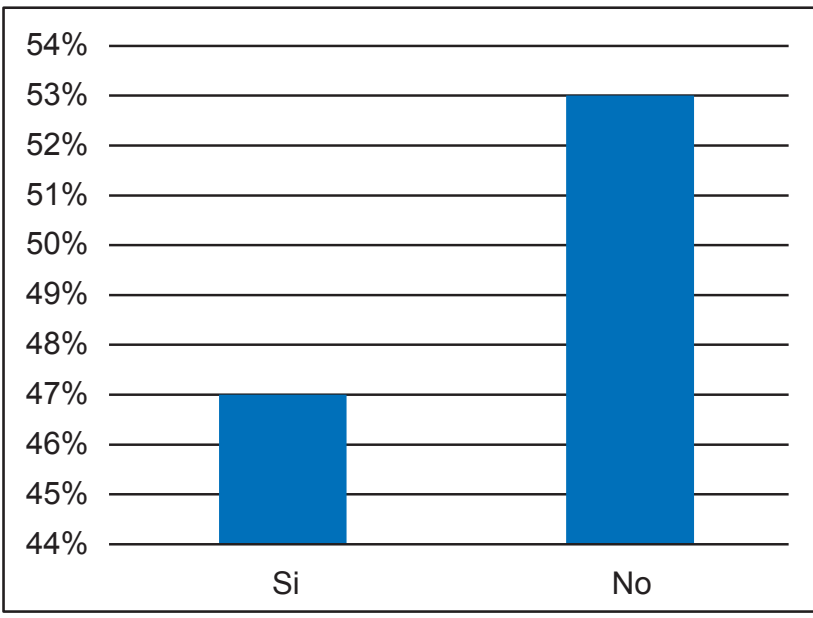

Fuente: Encuesta Asignatura Familiar II

Según los datos obtenidos el $86.6 \%$ de la población si sabe que es el abandono y el $13.4 \%$ no sabe.

Según los datos obtenidos, en las familias viven otros niños aparte de sus hijos. (Ver Tabla No. 2.)

Tabla No. 2: Familias en las que viven otros niños.

\begin{tabular}{|c|c|c|}
\hline $\begin{array}{c}\text { ¿En este hogar } \\
\text { habitan niños hijos } \\
\text { de un familiar? }\end{array}$ & $\begin{array}{c}\text { Frecuen- } \\
\text { cia }\end{array}$ & Porcentaje \\
\hline No & 86 & $57.72 \%$ \\
\hline Si & 63 & $42.28 \%$ \\
\hline Total & 149 & $100.00 \%$ \\
\hline
\end{tabular}

Fuente: Encuesta Asignatura Familiar II 
El $50.8 \%$ indicaron ser la abuela de los niños abandonados, $27 \%$ son tíos o tías de estos niños, el $7.9 \%$ no tienen parentesco con los niños, $6.35 \%$ el abuelo, el $4.78 \%$ hermanos, $3.17 \%$ primos. El $31.7 \%$ de los encuestados indicaron que los responsables de suplir las necesidades económicas del menor era la mamá, el $17.5 \%$ la abuela, $11.1 \%$ abuelo, $1.5 \%$ desconocido, $1.6 \%$ hermano, $6.4 \%$ tíos, $17.5 \%$ el padre, y $12.7 \%$ ambos padres. El $89.9 \%$ de las personas encuestadas indicaron que los menores nunca se quedan solos en el hogar y un $10.1 \%$ de los menores si quedan sin supervisión.

El $45 \%$ indicaron que demuestran el afecto hacia sus hijos por medio de caricias, $21.5 \%$ con abrazos, $19.5 \%$ dan palabras de motivación, los besos el $8.1 \%$, mientras que los obsequios el $5.4 \%$ y $0.5 \%$ no muestran un tipo de afecto.

El $55.7 \%$ de padres mencionaron que no utilizan palabras que afectan emocionalmente a su hijo, y $44.30 \%$ si utilizan palabras que afectan a sus hijos. En este estudio, (39) $26.17 \%$ de las familias entrevistadas refieren que los niños realizan actividades para generar ingresos. (Ver Tabla No. 3.)

Tabla No. 3: Actividades que realizan los niños para generar ingresos según la familia entrevistada.

\begin{tabular}{|c|c|c|}
\hline Actividades & Frecuencia & Porcentaje \\
\hline Construcción & 7 & $4.73 \%$ \\
\hline Jardinería & 4 & $2.70 \%$ \\
\hline Maquila & 1 & $0.68 \%$ \\
\hline Venta de comida & 4 & $2.7 \%$ \\
\hline Pulpería & 3 & $2.03 \%$ \\
\hline Venta ambulante & 20 & $13.51 \%$ \\
\hline Ninguno & 110 & $73.82 \%$ \\
\hline Total & 149 & $100.00 \%$ \\
\hline
\end{tabular}

Fuente: Encuesta Asignatura Familiar II

De las familias entrevistadas, el $53.85 \%$ indicaron que los niños realizan esas actividades solo los fines de semana, el $23.08 \%$ solo en la tarde, el $12.81 \%$ durante la mañana y el $10.26 \%$ todo el día.

\section{DISCUSIÓN}

El $47 \%$ ha observado niños mendigando en la Comunidad Garífuna de los cuales no se especifica la edad, ni el sexo. En Medellín, Colombia las encuestas aplicadas a 50 niños ejerciendo mendicidad, se estableció que el $71 \%$ de ellos tienen edades entre 5 y 10 años y $29 \%$ entre 11 y 14 años. El $40 \%$ de ellos son niños y el $60 \%$ son niñas. ${ }^{(10)}$

En el $42.28 \%$ de los hogares habían niños que no son hijos de las familias encuestadas y de estos el $50.79 \%$ estaban a cargo de las abuelas lo que contrasta con el estudio de la niñez Indígena y Afroamericana en donde el 50\% vive con ambos padres, $25 \%$ con la madre y el resto con otros familiares y no se menciona a la abuela. ${ }^{(2)}$ Esta cultura refleja el papel protagónico de la abuela en la estructuración de las familias y comunidades. La figura de la abuela como horcón familiar es una tradición, en el Caribe y en Estados Unidos, para las personas afro-descendientes. En aquellos hogares donde las mujeres jóvenes trabajan, las tías y las abuelas no sólo fungen como cuidadoras de los niños/as, además van proporcionándoles a la joven generación de una cultura con altos atributos afro-descendientes. ${ }^{(11)}$

De las familias que tiene sus hijos $39.60 \%$ indicaron que la abuela es quien cuida de los niños, $1.34 \%$ abuelo, $22,82 \%$ la mamá, $0.67 \%$ el padre, $14.09 \%$ tío o tía, $1.34 \%$ primos, $8.72 \%$ nadie, el $9.40 \%$ hermano o hermana y $2.01 \%$ desconocidos. Un estudio en Bogotá, Colombia indica que la mayoría de los padres, es decir un $38 \%$ prefiere dejar a sus hijos bajo el cuidado de las madres comunitarias cuando no pueden estar con ellos y el porcentaje más bajo, es decir $3 \%$ deja a los niños solos exponiéndolos a una situación de abandono. ${ }^{(12)}$

Los resultados de la investigación muestran que el $55.7 \%$ de personas mencionaron que no utilizan palabras que afectan emocionalmente a sus hijos y $44.3 \%$ si las utilizan. En Chile la violencia física grave hacia los niños ha disminuido pero cada vez más, los menores 
son víctimas de palabras insultantes, manipulación psicológica y descuido de sus necesidades afectivas por parte de sus padres. ${ }^{(13)}$

Con respecto al trabajo infantil, el $26.17 \%$ contestaron que los menores realizan este tipo de actividades para generar ingresos. La situación de trabajo infantil es común en países Centroamericanos como lo refleja la Encuesta Nacional de Trabajo Infantil y Adolescente en Nicaragua (ENTIA 2005), en donde 265,881 niñas, niños y adolescentes trabajan en edades de 5 a 17 siendo $70.8 \%$ varones y el 29.2 mujeres. ${ }^{(14)}$

El $13.51 \%$ realizan ventas ambulantes, $4.73 \%$ se dedica a la construcción, $2.7 \%$ a la jardinería y $2.7 \%$ la venta de comida, $2.03 \%$ a atender la pulpería de sus padres y $0.68 \%$ trabaja en maquila. Similares actividades pero con porcentajes más altos reporta la OTI en pueblos indígenas en Guatemala: Agricultura $70.5 \%$, industria $11.9 \%$, comercio $11.5 \%$, servicios $4.1 \%$ y otros $2.1 \%$. $^{(15)}$

La jornada laboral de estos niños es: $53.85 \%$ fines de semana, el $23.08 \%$ en la tarde, $12.82 \%$ en la mañana y el $10.25 \%$ todo el día. Lo que contrasta con un estudio exploratorio sobre el trabajo infantil que indica que la participación de los niños en actividades económicas o de trabajo doméstico es ocasional o temporal $91,2 \%$; se destaca el $47,8 \%$ que trabaja solo en vacaciones, el $11,1 \%$ fines de semana, $14,6 \%$ a veces, aunque sea período de clases. Sólo el 8,8\% de los niños se adhieren a un régimen de trabajo regular. ${ }^{(16)}$

Conclusión: en los resultados de la investigación se demuestra que si hay abandono, tanto físico como emocional en los niños de la comunidad Garífuna Travesía de Puerto Cortés, al igual que la mendicidad, aunque no se observan niños pidiendo a simple vista estos si rodean la comunidad o realizan esta actividad durante las fechas donde hay más turistas, el trabajo infantil no se ve muy marcado en la población, sin embargo los niños de las comunidades garífunas si realizan actividades económicas para generar ingresos en su hogar, como la venta de pan de coco, tabletas de coco, algunos en construcción, la mayoría de estos niños que trabajan también estudian.

Recomendaciones: Promover la importancia de la comunicación entre los padres y los niños abandonados. Promover la disminución del trabajo que realiza el niño, el cual interrumpe con las actividades académicas y de recreación.

Dar continuidad y apoyar las investigaciones venideras de las comunidades garífunas así como los demás grupos étnicos.

\section{REFERENCIAS BIBLIOGRÁFICAS}

1. Fondo de las Naciones Unidas para la Infancia. La infancia en Honduras: análisis de situación 2010. [Internet]. Tegucigalpa: UNICEF; 2011 [Citado 3 de abril 2016]. Disponible en: http://www.unicef. org/honduras/Sitan_-_Analisis_de_Situa cion-_Honduras_2010_2.pdf.

2. Observatorio de los Derechos de la Niñez. Niñez indígena y afrohondureña en la Republica de Honduras. [Internet].
Tegucigalpa: UNICEF; 2012 [Citado 3 abril 2016]. Disponible en: http://www. unicef. org/honduras/FINAL_DOCUMEN TO_COMPLETO_DE_NINEZ_INDIGE NA_A FROHONDÜRENA_2.pdf.

3. Chuquiana L. Mendicidad infantil [diapositiva en Internet]. 2013 [Citado 3 abril 2016]. Disponible en : http://es.slideshare. net/LeticiaChuquiana/mendicidad-infan til-24148704. 
4. Zumárraga F. Mendicidad Infantil. [Internet] Fernanda Zumarraga. sl. Mafer-zd87. [Citado 3 Abril 2016]. Disponible en: http://mafer-zd87.blogspot.com/2012/10/ mendicidad-infantil.html.

5. Wordpress.com. Que es el abandono infantil sus causas y síntomas. [Internet]. 2012 [Citado 3 abril 2016].

Disponible en: https://lanecesidaddeun hogar.wordpress.com/el-abandono-infan til/que-es-el-abandono-infantil-sus-cau sas -y-sintomas/.

6. Martín A. Trabajo infantil: tipos, causas, consecuencias. [Internet]. 2015. Lifeder.com [Citado 3 abril 2016]. Disponible en: http://www.lifeder.com/trabajo-infantil/.

7. Del olvido a la memoria: africanos y afromestizos en la historia colonial de Centroamérica [Internet]. San José: UNESCO; 2008.

Disponible en: http://unesdoc.unesco.or g/images/0018/001838/183846S. pdf.

8. Xplorhonduras, Grupos Garifuna de Honduras. [Internet]. Honduras: Xplorhonduras. (Citado 3 Abril 2016).

Disponible en: http://www.xplorhonduras. com/grupo-ga rifuna-de-honduras/.

9. Gonzáles S. Garífunas de Honduras. [Internet]. Honduras: Sixto Gonzales; 2011 [Citado 3 abril 2016] Disponible en: http://garifunashn.blogspot.com/.

10. Arroyave Moreno K, Rodríguez L, Arango I, Mesa A. Descubriendo la realidad que emerge tras la mendicidad infantil: La mendicidad de niños entre 5 a 14 años como factor de riesgo social en el asentamiento pacifico de la comuna 8 de la ciudad de Medellín. [Internet]. Corporación Universitaria Minuto de Dios; 2009. [Citado 16 Septiembre 2016]. Disponible en: http://repository.uniminuto.edu:8080/
jspui/bitstream/10656/3573/1/TTS_Arro yaveMorenoKarlaYurany_2010.pdf.

11. Alayon,A. Las contribuciones de las mujeres africanas a la cultura afro-americana. [Internet]. Cuba: Anabel Mitjans Alayón; 2013 [Citado el.....

Disponible en: https://negracubanatenia queser.com/2013/02/13/las-contribucio nes-de-las-mujeres-africanas-a-la-cultu ra-afro -americana-i/.

12. Sierra J. Tipos de abandono infantil en el sector de Buenavista en Bogotá [Internet]. Bogotá: Universidad de San Buenaventura; 2006. [citado 16 septiembre 2016]. Disponible en: http://bibliotecadigital.usb. edu.co/jspui/bitstream/10819/1222/1/Ti pos_abandono_infantil_\%20sector_ Sierra_2006.pdf.

13. Mohor D. Abandono emocional, el nuevo maltrato infantil. El Mercurio [internet] 2011 [citado 16 Septiembre 2016];

Disponible en: http://www.educativo.utal ca.cl/medios/educativo/articulosydoc/a bandono_emocional.pdf.

14. Fondo de las Naciones Unidas para la Infancia. Unicef en Nicaragua. [Internet] Managua: UNICEF.2008. [citado 17 septiembre 2016]. Disponible en: http://www. unicef.org/lac/UNICEF_en_Nicaragua.pdf.

15. Oficina Internacional del Trabajo. Trabajo infantil y pueblos indígenas: el caso de Guatemala. [Internet]. San José: OIT; 2006. [citado 17 septiembre 2016].

Disponible en: http://www.telefonica.com. gt/pro nino/assets/pdf/TrabajolnfantilPue blosIn digenasGuatemala.pdf.

16. Silva U, Damianovic N. Estudio exploratorio sobre trabajo infantil. [Internet]. sl. 1998 [citado 17 septiembre 2016].

Disponible en: http://www.dt.gob.cl/m/16 20/arti cles-62875_cuad_investig_1.pdf. 